\title{
Tracking Blade Tip Vortices for Numerical Flow Simulations of Hovering Rotorcraft
}

\author{
Paper ID\# 121
}

\begin{abstract}
Blade tip vortices generated by a helicopter rotor blade are a major source of rotor noise and airframe vibration. This occurs when a vortex passes closely by, and interacts with, a rotor blade. The accurate prediction of Blade Vortex Interaction (BVI) continues to be a challenge for Computational Fluid Dynamics (CFD) [1]. Though considerable research has been devoted to BVI noise reduction and experimental techniques for measuring the blade tip vortices in a wind tunnel, there are only a handful of post-processing tools available for extracting vortex core lines from CFD simulation data. In order to calculate the vortex core radius, most of these tools require the user to manually select a vortex core to perform the calculation. Furthermore, none of them provide the capability to track the growth of a vortex core, which is a measure of how quickly the vortex diffuses over time. This paper introduces an automated approach for tracking the core growth of a blade tip vortex from CFD simulations of rotorcraft in hover. The proposed approach offers an effective method for the quantification and visualization of blade tip vortices in helicopter rotor wakes.
\end{abstract}

Keywords: vortex core, feature extraction, CFD, numerical flow visualization

Index Terms: I.3.8 [Computer Graphics]: Applications

\section{INTRODUCTION}

Computational Fluid Dynamic (CFD) simulation of rotorcraft aeromechanics is a challenging time-dependent problem. There are many interesting flow phenomena that arise from the complex interactional aerodynamics of hover and forward flight. These include Blade Vortex Interaction (BVI), vortex pairing, vortex breakdown, vortex-wake interaction and the interaction of the vortex wake with the fuselage. These flows are typically multidisciplinary that combine moving body aerodynamics, deforming rotor blades, and a trim algorithm which determines the blade motion for a desired helicopter flight path. Rotor wake characteristics in a wind tunnel experiment are sometimes analysed using the Particle Imaging Velocimetry (PIV) technique, where flow field velocities can be measured [2]. Experimental data is often used to validate a CFD flow simulation. But regardless of how the rotorcraft flow fields are generated, via wind tunnel test or numerical simulations, it is important to utilize the results to better understand the complex nature of the flow

Our work has three main objectives: (1) code validation: compare CFD data with experimental results to gain confidence in the accuracy of the CFD simulation codes; (2) develop methods to provide greater detail and understanding of vortex properties from CFD simulations given CFD can provide a much higher resolution and more detail than acquired through an experiment; and (3) develop a vortex core visualization tool that domain scientists can apply to analyze their CFD data.

Presently there is a lack of software tools that can automatically detect a vortex, identify the evolving direction of the vortex filament, and then extract the circumferential velocity field and inferred vortex strength from CFD data on the plane perpendicular to the vortex filament. Thus, domain scientists needs to take some ad hoc steps towards an in-depth analysis of the vortex core, while this process can be time consuming and prone to user errors.

This paper presents an automated approach for tracking the blade tip vortex core growth, specifically, calculating radii of the core, from CFD data. Although there are a few commercial flow visualization packages for vortex core line extraction, none of them is able to automatically calculate vortex core radii without user intervention. In addition, most of them cannot track the growth of a vortex core. There has been some related work in automatic vortex core extraction, whereas tracking the vortex core growth remains an open problem [3].

The remainder of this paper is organized as follows. First, we describe the details of our novel method in Section 2. Next, we demonstrate some results of applying the proposed approach to three CFD simulation datasets in Section 3. Section 4 concludes this paper with a brief summary and outlook on future work.

\section{OUR APPROACH}

Our new approach consists of the following main steps:

(1) Generate a cutting plane (Section 2.1).

(2) Apply color map to the cutting plane to highlight vortex regions (Section 2.2).

(3) Cluster the on-plane points into vortex core clumps, and then locate the blade tip vortex (Section 2.3).

(4) Calculate the radius of the blade tip vortex, and then track the growth of the vortex core (Section 2.4).

\subsection{Cutting Plane}

During wind tunnel tests, velocity components of a flow field are sometimes measured on a stationary plane using PIV techniques. Our method is motivated by the fact that CFD provides much more information than direct measurement. We propose to emulate the experimental PIV plane with a Y-Z cutting plane, which is positioned at $\mathrm{x}=0$. While the cutting plane is represented by a Cartesian grid, the velocity vector field on the cutting plane is obtained via $3 \mathrm{D}$ interpolation of the input flow field. The size of the cutting plane, specified by the user, determines the coverage of the wake field. Figure 1 shows a cutting plane relative to the hub and blades of an XV-15 3-bladed rotor system without the fuselage. Our method assumes that the rotor hub is parallel to the positive $z$ axis and the cutting plane will be rotated about the hub ( $z$ axis) in subsequent steps.

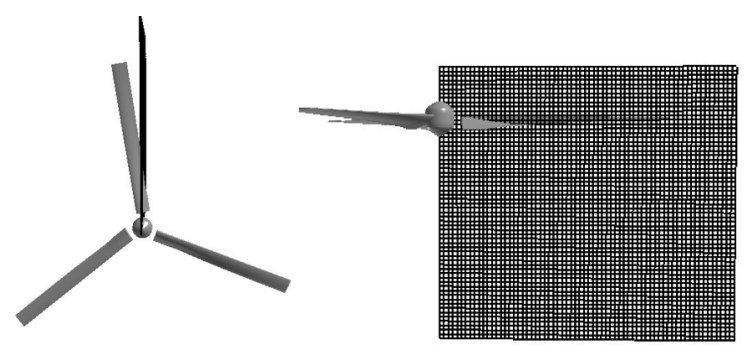

Figure 1: Top and side views of the cutting plane with respect to the hub and blades of an XV-15 rotor system. 


\subsection{Scaled Q-Criterion Contours}

In a rotorcraft flow field, the vortex shed from a blade tip diffuses as the blade rotates about the rotor hub. Our goal is to track the core radius of the blade tip vortex in an instantaneous rotorcraft flow field. As we first need to locate the blade tip vortex, a

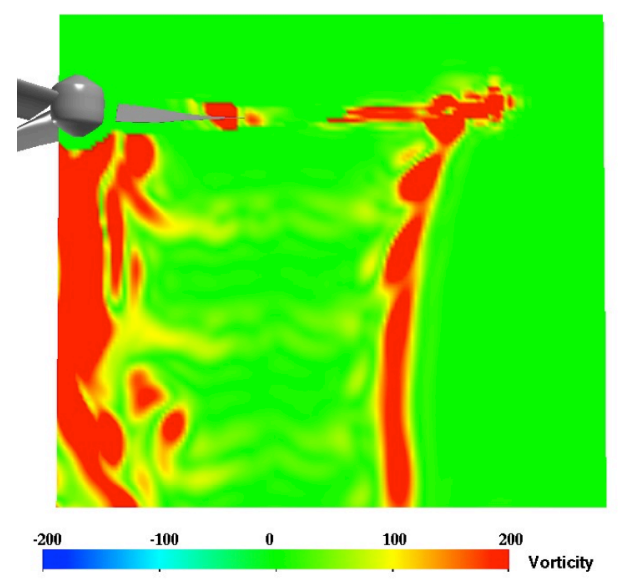

Figure 2: Cutting plane colored by vorticity.

popular method for locating vortices is to reveal the flow structure using contour maps of a scalar quantity. Figure 2 shows vorticity contours on a cutting plane of a rotorcraft flow field in hover mode. Several vortices are depicted, whereas their boundaries are not delineated clearly. How well the blade tip vortex boundary is revealed depends on threshold values selected in the contour color map. A common challenge of displaying contour maps of a scalar quantity consists in the specification of appropriate threshold values. A small value may reveal excessive flow structures while a large one may suppress important features.

We propose to use the scaled Q-criterion (or $Q_{s}$ for short), which is non-dimensional and defined as follows:

$$
Q_{s}=\frac{1}{2}\left(\frac{\|\Omega\|^{2}}{\|S\|^{2}}-1\right)
$$

where $\Omega$ is the rotation rate tensor and $S$ is the strain rate tensor. The advantage of using $Q_{s}$ as a scalar quantity for creating contours is that a threshold of 1 gives a good approximation to the vortex core boundary. Specifically, any point with $\mathrm{Q}_{\mathrm{s}}>=1$ tends to fall within a vortex region [4]. Figure 3 shows the same cutting plane as in Fig. 2, but with a color map based on $\mathrm{Q}_{\mathrm{s}}$. Note that the vortex core regions in Figure 3 are more prominent than in Fig. 2.

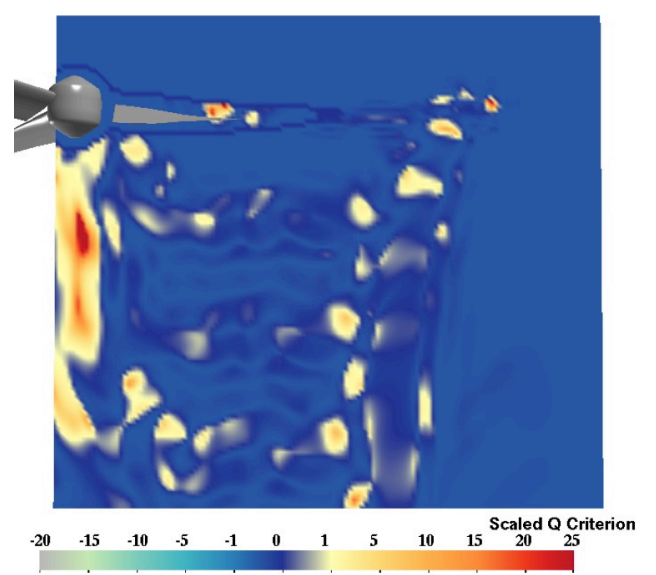

Figure 3: Cutting plane colored by scaled $Q$-criterion $\left(Q_{S}\right)$.

\subsection{Blade Tip Vortex Clump Calculation}

Even with a good depiction of vortex regions on a cutting plane as in Figure 3, we do not yet know the number of vortex regions and the point-region correspondence. To identify vortex regions, we propose to cluster the colored contours on the cutting plane into

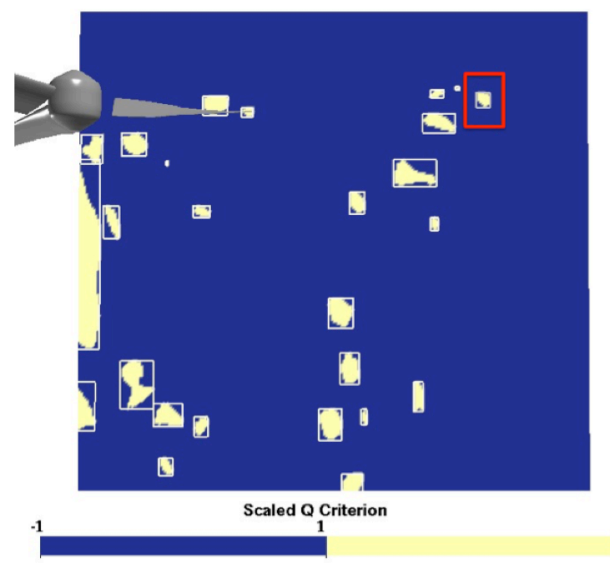

Figure 4: Vortex regions enclosed by white boxes. The blade tip vortex is inside the ROI, which is denoted by the red box.

different clumps, with each clump representing a vortex region. This method works by using a color map with 2 color bands, rather than a traditional continuous map employed in Figs. 2 and 3. Figure 4 shows the cutting plane with a two-banded color map (i.e., blue and light beige). Now vortex core regions can be easily discerned, with each vortex clump bounded by a white box

To locate the blade tip vortex, we search for the clump that falls within a region of interest (ROI) centered around the blade tip. In Fig. 4, the ROI is indicated by the red box. As shown in Fig. 4, the clump inside the ROI red box is the blade tip vortex that will be analyzed further in the next section.

\subsection{Vortex Core Growth Tracking}

Once we locate the clump used for representing the blade tip vortex on the cutting plane, the next step is to determine the vortex core center inside this clump. We search for the point with the maximum $Q_{s}$ inside the tip clump. There are many wellknown methods in the literature for vortex core detection, such as those based on predictor-corrector [5], velocity gradient [6], and parallel vectors $[7,8]$. Our approach is based on a simple yet effective one that adopts a scalar quantity to locate the vortex core center. In fact, our tests show that the local minimum pressure [5] and the local maximum vorticity [9] methods do not exhibit a significant difference in vortex core radius calculation.

Next, we calculate the vortex core radius of the tip clump using the core center found above. A typical method is to extract a cross-flow velocity profile on the plane perpendicular to the vortex filament. Since the vortex core is not necessarily perpendicular to the cutting plane, we need to determine the vortex filament direction. We rotate the cutting plane about the rotor hub with a small azimuthal increment of $5^{\circ}$. We then repeat the aforementioned procedure to calculate the core center of the new cutting plane - the rotated version. Let $P_{i-1}$ and $P_{i}$ be the core centers found in the original and the new cutting planes, respectively. We assume line $P_{i-1} P_{i}$ to be aligned with the vortex filament direction.

Figure 5 shows a cross-flow profile plot of the u-velocity component at the blade tip vortex center, which is inside the red box shown in Fig. 4. The local minimum speed occurs at $\mathrm{w}$ $\sim=144 \mathrm{~mm}$ and the local maximum speed at $\mathrm{w} \sim=152 \mathrm{~mm}$. The 


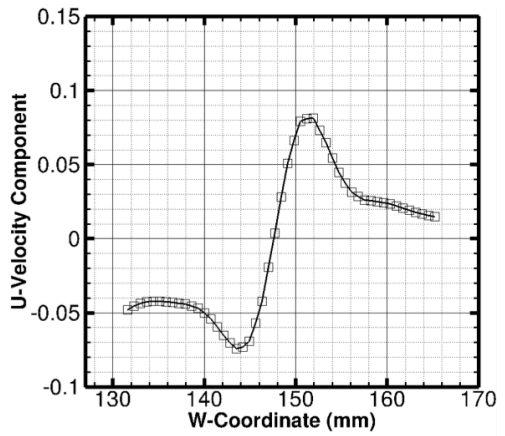

Figure 5: The cross-flow velocity profile at the blade tip vortex center.

core diameter is equal to the distance between these two positions and the core radius is half of the diameter. Our approach calculates the cross-flow profile along two local orthogonal axes (e.g., $u$ and $\mathrm{w}$ axes). The vortex core radius is then the average of two core radii from the two cross-flow profiles. The reason for generating the two profiles is that the extracted vortex core center (either via the local maximum vorticity or local minimum pressure) may not be precisely at the core center. Furthermore, in a rotorcraft wake, there is a dominant rotational flow motion due to the rotating blades. Our approach calculates the average velocity of all points inside the blade tip clump, and then subtracts the average velocity from all velocity vectors prior to calculating the cross-flow profiles.

By keeping track of the tip vortex clump as the cutting plane rotates about the rotor hub, the blade tip vortex core growth can be captured until the algorithm cannot locate the tip clump due to vortex diffusion or until the vortex reaches the boundary of the cutting plane. Since the blade tip vortex clump is obtained separately as the cutting plane rotates, no correlation is readily available between any two consecutive cutting planes. Our algorithm establishes this relationship such that the tip vortex clump can be tracked as the cutting plane rotates about the rotor hub.

\section{Results And Discussions}

We apply our novel blade tip vortex tracking method to three flow simulation datasets resulting from a Navier-Stokes grid resolution study for an isolated V22 TRAM rotor with simplified hub in hover [10]. The flow simulation data were generated by the OVERFLOW simulation code [11]. Using an overset gridding scheme [12], the hub and its three blades were embedded in a uniformly spaced Cartesian background grid. For the grid resolution study, various levels of refined Cartesian grids were embedded into the background to better resolve the rotor blade vortices. Figure 6 gives a view of the baseline grid system (on which the first dataset is based, without grid adaptation) on the plane of $\mathrm{z}=0$, with $\Delta \mathrm{S}_{1}=2.2$ inches ( $10 \%$ of the rotor blade tipchord) as the grid spacing. This dataset consists of 120 million grid points and is referred to as the dataset with a coarse grid resolution.

Figure 7 shows blade tip vortex lines formed by connecting the core centers extracted from the first dataset, with the black line created via the local maximum scaled Q-criterion and the red line via the local minimum pressure. As expected, there are only a few noticeable differences between these two vortex lines. A plot of the vortex core growth is shown in Fig. 8 for the core radii calculated using the local maximum $\mathrm{Q}_{\mathrm{s}}$ (green circles) and local minimum pressure (cyan circles) methods, respectively. Due to the low resolution of the baseline grid, there is a considerable variation of the vortex core radius, and our method was only able to track the blade tip vortex up to a wake age of $370^{\circ}$. Wake age

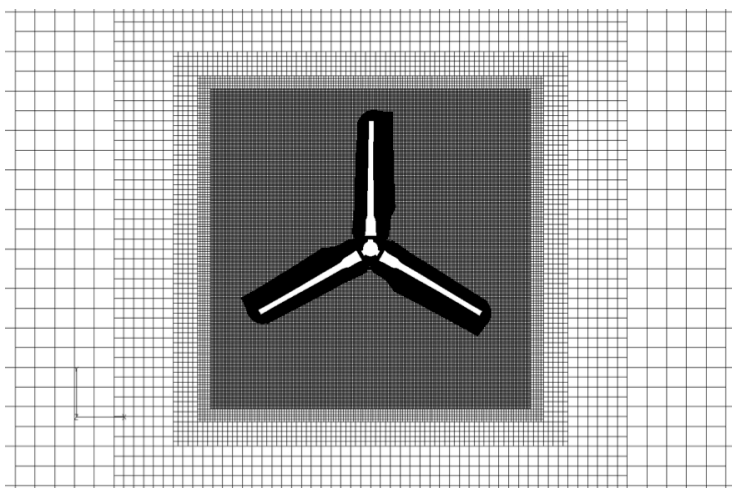

Figure 6: Top view of the uniformly spaced background grid enclosing the rotor hub and blades (grid spacing $=2.2$ inches).

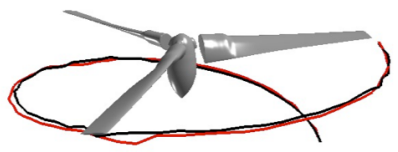

Figure 7: Vortex core lines generated using the local maximum Qs (black curve) and the local minimum pressure (red curve) using dataset \#1 (coarse grid resolution).

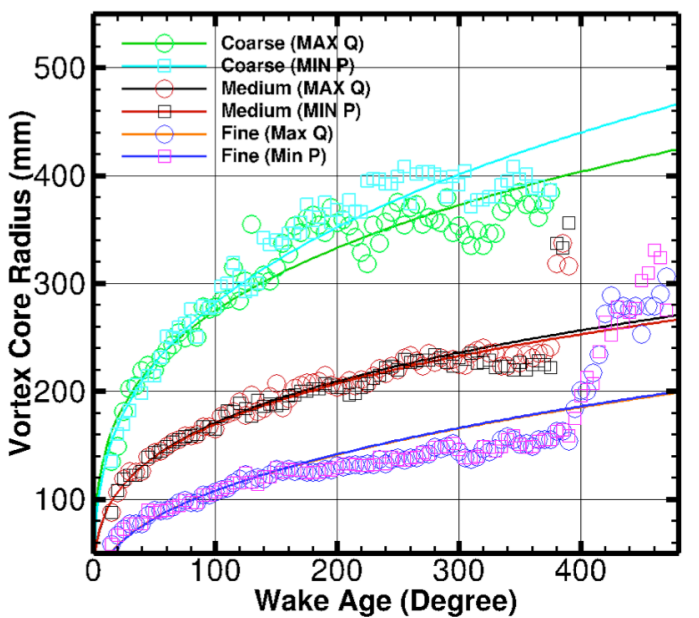

Figure 8: Vortex core growth computed using all three datasets.

is a measure of how many degrees the cutting plane has rotated from the blade. On a Linux Redhat system with 38 GB RAM running on a single $3.6 \mathrm{GHz}$ processor, it took approximately 30 seconds to extract the vortex core and track its growth. Without using our new approach, it could take several hours to extract the core radius either manually or by some ad-hoc method with a post-processing CFD tool such as FieldView or TecPlot.

The second dataset of the grid resolution study is based on a refined version of the baseline grid, with 164 million points and $\Delta S_{2}=\Delta S_{1} / 2=1.1$ inches as the grid spacing. Figure 9 shows two vortex core lines extracted by the local maximum $Q_{s}$ and the local minimum pressure methods, respectively, from the second dataset. The vortex core growth reflected by this dataset is shown in Fig. 8. As expected, the extracted vortex core radii from this dataset (which we refer to as medium in Fig. 8) are less than those from 


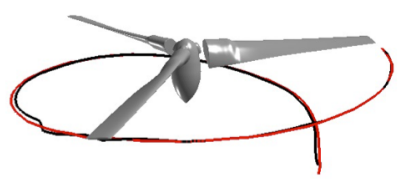

Figure 9: Vortex core lines generated by using the local maximum $Q_{s}$ (black curve) and the local minimum pressure (red curve) using dataset \#2 (medium grid resolution).

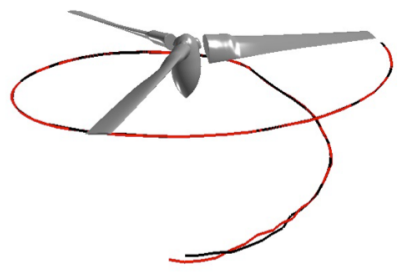

Figure 10: The vortex core lines generated using the local maximum $Q_{s}$ (black curve) and local minimum pressure (red curve) methods, respectively, from dataset \#3 (fine grid).

the first dataset due to the increased grid resolution. The time cost for tracking the vortex core growth is approximately 172 seconds. With this medium grid resolution, our approach was able to track the blade tip vortex slightly longer (wake age at $\theta=390^{\circ}$ ). But there is no significant difference between the vortex lines shown in Figs. 7 and 9.

The third dataset of the study is based on another refined version of the baseline grid, specifically with 486 million points and $\Delta S_{3}=\Delta S_{1} / 4=0.55$ inches as the grid spacing. A plot of vortex core lines extracted from this dataset is shown in Fig. 10. With this higher level of refinement, our approach was able to track the blade tip vortex up to $\theta=470^{\circ}$, and the vortex core lines are longer in the refined-grid rotor wake field than those shown in Figs. 7 and 9. Fig. 11 shows the two cross-flow profiles used to compute the vortex core radius for the cutting plane at $25^{\circ}$. As shown in Fig. 11, the vortex radii computed from the $\mathrm{w}$ and $\mathrm{u}$ profiles are $74 \mathrm{~mm}$ and $70 \mathrm{~mm}$, respectively. The average radius is $72 \mathrm{~mm}$. The vortex core growth obtained from the third dataset with the finer grid is also shown in Fig. 12, where the vortex core radii are less than those computed from the coarse and medium grid resolutions. The increase of vortex core size at $\theta=400^{\circ}$ is due to the vortex passing into a coarse-grid region. The execution time for calculating the vortex core growth is approximately 278 seconds.

A typical approach for analysing the vortex core growth is to plot the Normalized Vortex Core Diameter (NVCD), instead of the actual radius, which is the core diameter divided by the rotor blade tip-chord. Figure 11 depicts the NVCD values resulting from the three datasets, while data measured from an experiment [12] are also shown for comparison. Among the three cases, the vortex core growth based on the finest grid (for the third dataset) shows the closest agreement with the experiment.

\section{Conclusion}

We have presented an effective as well as efficient method for tracking the vortex core growth in CFD simulation of rotorcraft flows. It is able to track the vortex core growth automatically in a few minutes, much faster than a manual approach, which usually takes several hours. Currently, the user needs to specify an ROI for detecting the blade tip vortex. In future work, we will enhance the proposed algorithm with automatic intelligent ROI selection. As we are now developing the code, we will release a software tool based on the proposed method for rotorcraft applications. In addition, we plan to extend the algorithm to non-rotorcraft flow simulations, for which a non-rotational transformation may be applied to the cutting plane. Furthermore, the rotation axis could be chosen to be non-parallel to any of the coordinate axes.

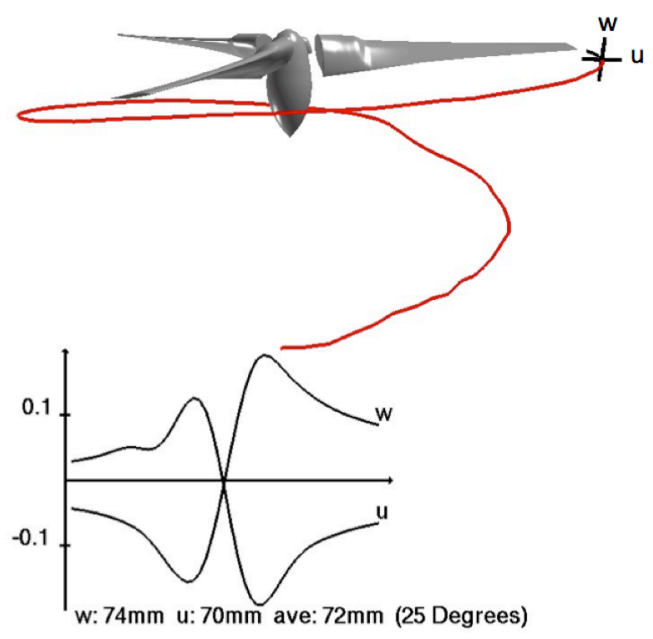

Figure 11: Two cross-flow profiles (lower left) at the vortex core center represented by the cross with the $u$ and $w$ axis labels. The red vortex core line is generated from dataset \#3.

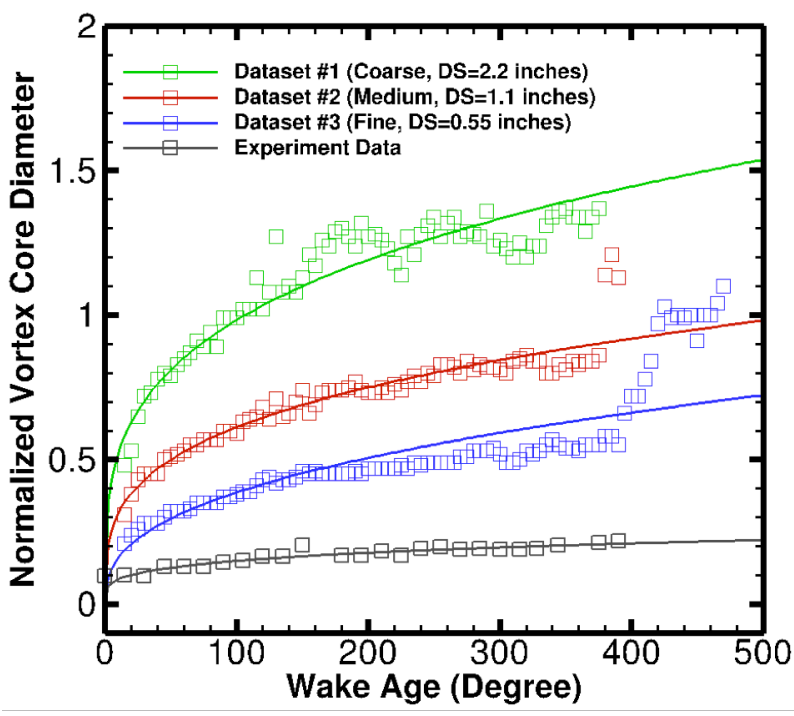

Figure 12: Comparison of the three datasets (based on different grids) along with the experiment result in the vortex core growth by Normalized Vortex Core Diameter (NVCD). 


\section{References}

[1] Y. Tanabe, S. Saito, K. Takasaki, and H. Fujita. Numerical study of blade-vortex interaction (BVI) noise capturing. IJASS, volume 9 , number 2, pages 48-57, November 2008.

[2] G. Yamauchi, A. Wadcock, W. Johnson, and M. Ramasamy, Wind Tunnel Measurements of full-scale UH-60A rotor tip vortices, Proceedings of the American Helicopter Society $68^{\text {th }}$ Annual Forum, 2012.

[3] D. Kao, J. Ahmad, and T. Holst, Visualization and Quantification of Rotor Tip Vortices in Helicopter Flows, AIAA Paper 2015-1369, 2015.

[4] S. Kamkar, A. Wissink, V. Sankaran, and A. Jameson, Featuredriven Cartesian Adaptive Mesh Refinement for Vortex-Dominated Flows," Computational Physics, Vol. 230, No. 16, pp. 6271-6298, 2011.

[5] D. Banks and B. Singer. A Predictor-Corrector Technique for Visualizing Unsteady Flow, Proceedings of IEEE Visualization '94, pp. 132-139.

[6] D. Sujudi and R. Haimes, Identification of Swirling Flow in 3-D Vector Fields, $12^{\text {th }}$ AIAA Computational Fluid Dynamics Conference, AIAA Paper 95-1715, 1995.

[7] R. Peikert and M. Roth, The "parallel-vectors" operator - a vector field visualization primitive. Proceedings of IEEE Visualization '99, pp. 263-270

[8] R. Fuchs, R. Peikert, H. Hauser, F. Sadlo, and P. Muigg, Parallel Vectors Criteria for Unsteady Vortices, IEEE Transactions on Visualization and Computer Graphics, Vol. 14, No. 3, pp. 615-626, May/June 2008.

[9] R. Strawn, D. Kenwright, and J. Ahmad, Computer Visualization of Vortex Wake Systems, AIAA Journal, volume 37, number 4, 1999.

[10] T. Holst and T. Pulliam, Overset Solution Adaptive Grid Approach Applied to Hovering Rotorcraft Flows, 27 $7^{\text {th }}$ AIAA Applied Aerodynamics Conference, AIAA Paper 09-3519, 2009.

[11] R. Nichols, R. Tramel, and P. Buning, Solver and turbulence model upgrades to OVERFLOW 2 for unsteady and high-speed applications, AIAA paper 2006-2824, 2006.

[12] W. Chan, Developments in strategies and software tools for overset structured grid generation and connectivity, AIAA paper 2011-3370, $29^{\text {th }}$ AIAA Computational Fluid Dynamics Conference, 2011.

[13] K. McAlister and J. Heineck, Measurements of the Early Development of Trailing Vorticity from a Rotor, NASA/TP-2002211848, AFDD/TR-02-A001, 2002. 